Introduction: Childhood obesity is a growing epidemic in many parts of the world. This is a major public health problem because obesity often tracks into adulthood, with many complications. During the last two decades, the prevalence of childhood obesity has been estimated in several countries. In Belgium, data are available from some parts of the country but no recent data exist for the province of Liege. The objective of the study is to assess the current prevalence of overweight and obesity in schoolchildren from the province of Liege.

Method: A retrospective study was conducted from the school health records of 1403 children monitored in 2005-2006 by school health centres in the Province of Liege. Height and weight, as part of routine child health monitoring, have been measured in light clothing by trained nurses. Complementary data such as physical activity were also recorded.

Results: The mean age of the population studied was 7 years $(2 \cdot 26-14 \cdot 6$ years). Using the IOTF definition, the prevalence of overweight (including obesity) was $16.6 \%$ for girls and $15.9 \%$ for boys, and the prevalence of obesity was $3 \cdot 96 \%$ and $4 \cdot 44 \%$, respectively. Overweight is increasing with age, particularly in girls between 5 and 8 years of age.

Conclusions: In comparison with other European countries, the prevalence of overweight and obesity in the Belgian province of Liege is intermediate, between the high prevalence in Southern Europe and the relatively lower prevalence in Northern Europe. It will be important to follow the situation in order to adjust prevention programmes.

\title{
05 - Abdominal obesity in children aged 12 years: a cross-sectional study in the Belgian province of Liege
}

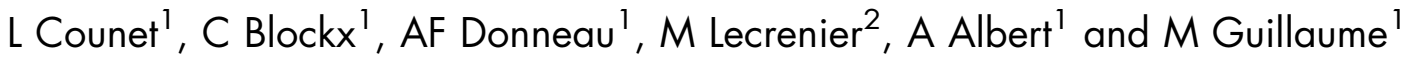 \\ ${ }^{1}$ University of Liege, School of Public Health: ${ }^{2}$ Province of Liege, Direction of the School Health Centres
}

Introduction: Abdominal obesity is a major component of metabolic syndrome (MS), a cluster of risk factors for CVD. It can already be detected in late childhood and adolescence, which is important in terms of prevention. In populationbased study, waist circumference (WC) is considered as a reliable indicator of abdominal obesity, which estimates the risk of developing MS. The objective of the study is to determine the prevalence of abdominal obesity in children aged 12 years in the province of Liege.

Method and population studied: A cross-sectional study was conducted in 2008-2009 including 784 children selected at random among the population of the school health centres, Province of Liege. WC was measured by trained nurses and complementary data were recorded. To assess abdominal obesity, percentiles of WC have to be used. No specific percentile curves for Belgian adolescents are available. Therefore, percentile curves from other countries were used.

Results: The mean age of the studied population was 11.5 (SD 0.5 ) years. Prevalence of abdominal obesity varied between $15 \cdot 4 \%$ according to the US percentile curves and $48 \cdot 4 \%$ with the percentile curves of Great Britain and was significantly higher in girls than in boys. There was a significant association between WC, age, BMI and professional status of the mother in both sexes.

Conclusions: Abdominal obesity in adolescents seems to represent a significant public health problem in the province of Liege. No data are available to compare with other Belgian regions. To better understand the situation, the development of Belgian-specific reference curves is needed.

\section{6 - Overweight and obesity in a representative sample of schoolchildren - exploring the urban-rural gradient in Sweden}

\author{
A Sjoberg ${ }^{1}$, L Moraeus ${ }^{1}$, A Yngve ${ }^{2,3}$, E Poortvliet $^{2}$, U Al-Ansari ${ }^{2}$ and L Lissner ${ }^{1}$ \\ 'Department of Public Health and Community Medicine, Public Health Epidemiology Unit, Sahlgrenska Academy, \\ University of Gothenburg, Sweden: ${ }^{2}$ Department of Biosciences and Nutrition, Karolinska Institutet, Sweden: \\ ${ }^{3}$ Faculty of Health, Nutrition and Management, Akershus University College, Norway
}

